\title{
Rhabdoid Meningioma-An Uncommon and Aggressive Variant
}

Asaranti Kar*, Gayatri Rath, Lity Mohanty, Pradip Behera, Sitaram Mohapatro, Anasuya Lenka and Shiva Soumendra Sahu

Department of Pathology, S.C.B.Medical College, Cuttack, Odisha, India

\begin{abstract}
Rhabdoid meningioma is a rare subtype of meningiomas accounting for $1-3 \%$ of all intracranial meningiomas and classified as WHO Grade III tumor. It has an aggressive course and needs to be treated by both surgery and radiotherapy. Here we present a rare case of rhabdoid meningioma in a 24 year old lady with complaints of focal seizures, paresis and diplopia. CT \& MRI showed a mass attached to dura in left posterosuperior frontoparietal region with peripheral edema. Histopathology showed large tumor cells arranged in sheets, whorls and papillary pattern. The patient was treated by surgery followed by radiation. This is reported to aware the surgical pathologists to keep this subtype as a differential diagnosis while interpreting any meningioma as it has an aggressive course.
\end{abstract}

Keywords: Intracranial; Meningioma; Rhabdoid

\section{Introduction}

Meningiomas are tumors of meningothelial origin and are mostly slow growing tumors having a wide range of histological appearances. Some subtypes however are recognized in WHO classification to display aggressive behavior including atypical, clear cell, chordoid meningiomas (WHO grade II) and papillary and anaplastic variants as WHO grade III. Recently, rhabdoid meningiomas have been added to the grade III subtype. These anaplastic meningiomas represent $2-3 \%$ of all meningiomas [1]. These are associated with rapid growth, brain invasion and frequent recurrences. Rhabdoid meningiomas were described for the first time in 1998 as an unusual variant with increased proliferative activity. Later, in 2000, these tumors were included in the revised WHO classification of CNS tumors as an aggressive meningioma corresponding to WHO grade III.

We report this case because of its unusual presentation at an earlier age which should be kept in mind while dealing with meningiomas.

\section{Case History}

A 24 year old female attended the Medicine OPD of S.C.B. Medical College, Cuttack with the complaints of focal seizure; with secondary generalization (1-2 episodes a month) for last 2 yrs. Weakness of right lower limb occurred followed by upper limb for last 1 year. Inability to walk without support for last 3 months. Blurring of vision and diplopia on right lateral gaze was noticed for last 15 days. There was no history of trauma, fever, convulsion, and diminution of hearing, difficulty in chewing or swallowing. Patient was non-diabetic, non-hypertensive and not a case of tuberculosis.

With these complaints, she was referred to the Neurosurgery Dept. for further neurological investigations and management. In general examination, patient was found conscious, well oriented, judgment was normal, recent memory normal, but remote memory and calculation were impaired with dyslexia $(+)$, finger agnosia $(+)$.

Visual acuity was bilateral 6 / 36, fundus showed papilledema, right lateral rectus palsy, right facial palsy (upper motor neuron) grade -2, right hemiparesis (3/5 in upper limb, $2 / 5$ in lower limb) with DTR exaggerated on right side, plantar $\downarrow \uparrow$, Sensory - primary modality intact, cortical modality (stereognosis, 2 pt discrimination graphesthesia) lost on right side. No cerebellar or meningeal sign was seen. MRI revealed left parietal superior posterior frontal interface complex signal altered, space occupying lesion with profound perifocal edema and mild mass effect, apparently representing tumor bleed. (Figure 1) CT scan showed the mass fixed to dura with indistinct tumoral margins, a mushrooming growth pattern characterized by multinodular projection from the main mass and edema of the neighboring brain (Figure 2). Ultrasonography of abdomen and pelvis did not reveal any abnormality.

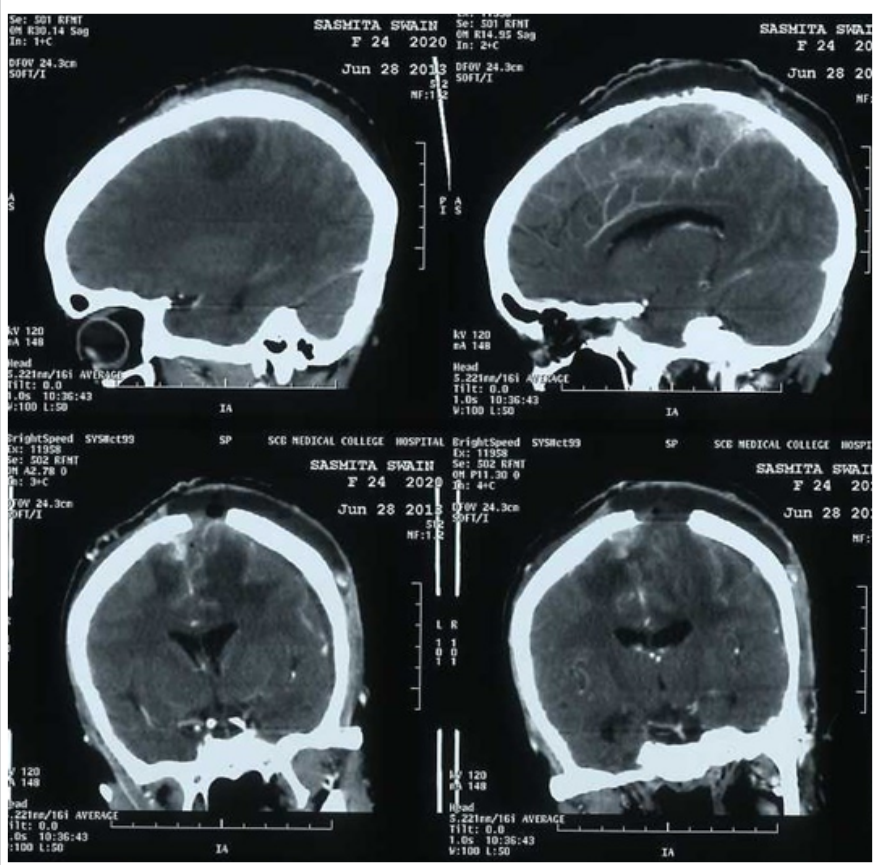

Figure 1: MRI showing lesion in left frontoparietal region.

${ }^{*}$ Corresponding author: Asaranti Kar, Qrs. No.JO-1, S.C.B.Medical College Campus, Cuttack, Odisha-753007, India, Tel: 09437170442; E-mail: asarantikar@yahoo.co.in

Received July 29, 2013; Accepted October 21, 2013; Published October 23, 2013

Citation: Kar A, Rath G, Mohanty L, Behera P, Mohapatro S, et al. (2013) Rhabdoid Meningioma-An Uncommon and Aggressive Variant. J Cancer Sci Ther 5: 343-345. doi:10.4172/1948-5956.1000225

Copyright: @ $2013 \mathrm{Kar}$ A, et al. This is an open-access article distributed under the terms of the Creative Commons Attribution License, which permits unrestricted use, distribution, and reproduction in any medium, provided the original author and source are credited. 


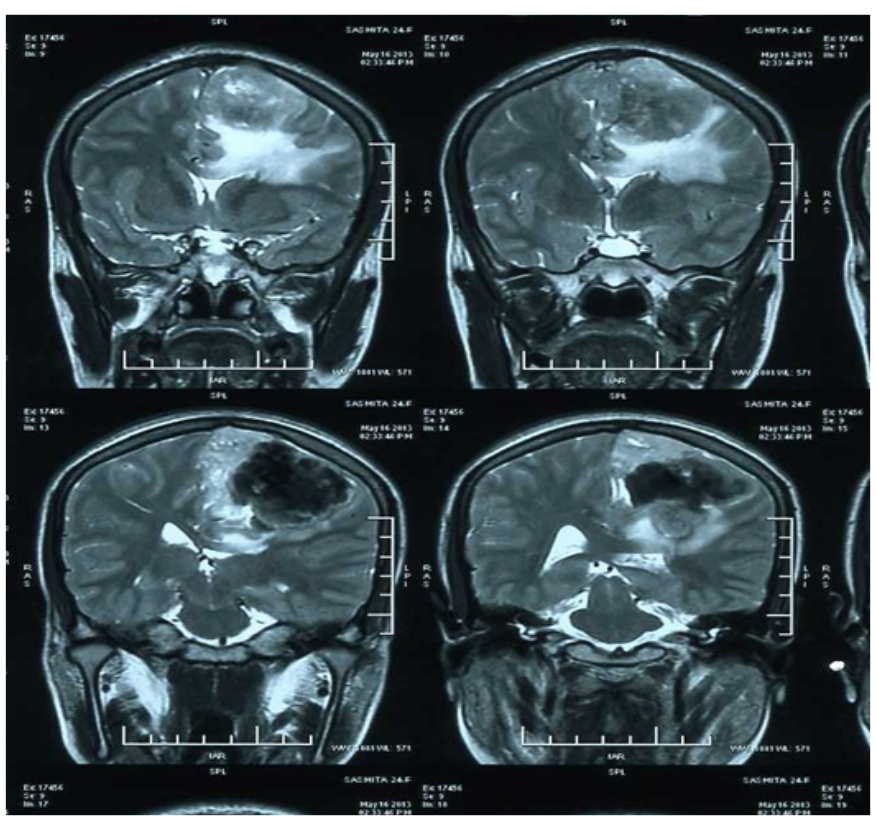

Figure 2: CT scan of the mass fixed to dura with peripheral oedema.

\section{Operative Findings}

Left frontoparietal skin incision was given just crossing the midline and true bone flap craniotomy was done in supine position. The tumor was adherent to dura and bone posteromedially. Dura was reflected medially. A firm to hard, moderately vascular mass attached to falx and dura was seen and was excised in multiple large pieces. The posterior aspect of the mass was cystic with hemorrhage, inside. The tumor which was infiltrating the mid falx and had gone to opposite side, was excised by incising the falx anteroposteriorly. Hemostasis was maintained. Water tight dural closure was done with duroplasty with G-patch. Normal bone fixed with polyamide suture and drilling the bone. The wound was closed in layers.

Grossly, multiple bits of firm irregular grayish white tissues together measuring about $7 \times 6 \times 4 \mathrm{cms}$ were received. The mass was firm to hard .Cut section was whitish and solid (Figure 3).

Micro section showed tumor cells arranged in sheets, small loose clusters with focal papillary pattern (Figure 4). Significant foci of large, round to polygonal cells, with abundant pale eosinophilic cytoplasm, central to eccentrically placed vesicular nuclei and prominent nucleoli with mild atypia (rhabdoid cells) were appreciated and intranuclear cytoplasmic pseudoinclusions were present at places (Figure 5). There were 5-10 mitoses for $10 \mathrm{HPF}$ and a proliferation rate (ki 67) of about 5\%. Immunotristochemistry showed positivity for epithelial membrane antigen and Vimentin. Corroborating the classical clinical presentations, the CT and MRI findings and the characteristic histomorphology, a final diagnosis of rhabdoid meningioma was made.

\section{Discussion}

The term rhabdoid morphology in tumor refers to the characteristic resemblance of the cells to a rhabdomyoblast without true skeletal muscle differentiation. The term malignant rhabdoid tumor (MRT) was first used to describe a distinctive pediatric renal tumor [2]. Subsequently, the term was applied to similar extra renal pediatric tumor (extra renal MRT) including atypical teratoid / rhabdoid tumor
(AT/RT) of the CNS [3]. These tumors commonly show mutation of the INI1 gene on 22.q11.2. Tumors with different histogenesis including carcinomas, sarcomas, gliomas and melanomas (composite extra renal rhabdoid tumor or ERT) also may show rhabdoid phenotype, but they don't show 22q11.2 deletion [4]. But an important feature, common to all rhabdoid tumors is aggressive behavior and poor outcomes irrespective of their histogenesis. In 1998, Kepes et al and Perry et al described the first 2 series of meningiomas with rhabdoid transformation $[5,6]$. These meningiomas often recur, invade brain and lead to extra cranial metastasis.

Besides the rhabdoid cells , cytoarchitectural features of atypical meningioma like more than or equal to 4 mitoses per 10 high power fields, high cellularity, sheeting architecture, nuclear atypia, necrosis are often seen in most cases [7]. A histological association with papillary subtype of meningioma (which is also aggressive) has been noted and was also appreciated in our case [8]. Clinically these tumors arise in elderly persons with equal incidences in male and female. Most patients present with neurological features like hemiparesis, diplopia, vomiting etc. The present case was noted in a 24 year old female who presented with chief complaints of focal seizure, paresis and diplopia.

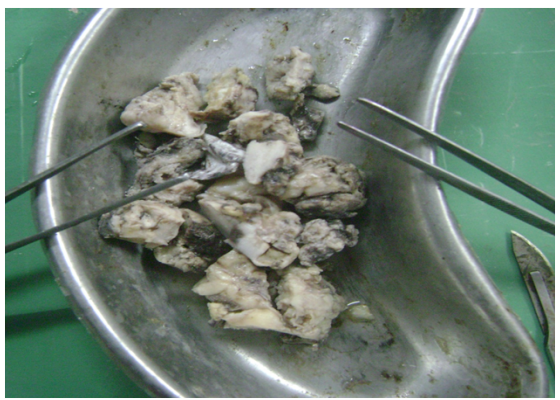

Figure 3: Gross received in multiple greyish white masses.

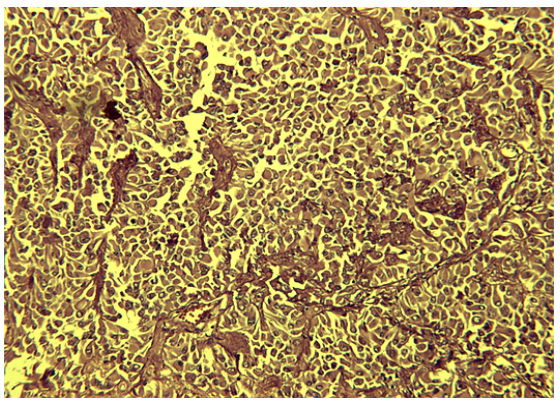

Figure 4: Histopathology of rhabdoid meningioma H\&Ex100

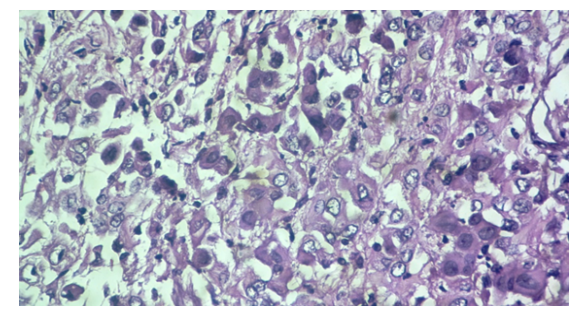

Figure 5:Large tumor cells with nuclear pseudo inclusion \& nuclear grooving H\&Ex400. 
Citation: Kar A, Rath G, Mohanty L, Behera P, Mohapatro S, et al. (2013) Rhabdoid Meningioma-An Uncommon and Aggressive Variant. J Cancer Sci Ther 5: 343-345. doi:10.4172/1948-5956.1000225

The differential diagnosis includes metastatic carcinoma, melanoma, glioma, sarcoma and AT /RT. Diagnosis however depends on finding evidences of meningothelial differentiation either by light microscope (like whorls, intranuclear pseudoinclusions, nuclear grooving), immuno histochemistry findings of expression of epithelial membrane antigens, vimentin, progesterone receptor positivity or electron microscopic findings of interdigitating cytoplasmic membrane, intercellular junction etc. In contrast to carcinomas, malignant meningiomas usually exhibit extensive EMA \& weak cytokeratin. Ki - 67 (MIB-1) is a valuable guide to malignancy in meningioma. The mean labeling index for such tumors is $14.7 \% \pm 9.8 \%$. In our case, it was about $5 \%$.

The final diagnosis of rhabdoid meningioma in the present case was rendered due to clinical and radiological findings in addition to presence of focal whorling, intranuclear pseudoinclusion/ nuclear grooving with classical rhabdoid morphology and demonstration of EMA positivity in immunohistochemistry.

Surgery is the sole modality of treatment followed by the conventional fractionated radiation therapy, usually to doses ranging from 50 to 60 Gy of radiation via conventional fractionation and delivery techniques. Currently, most investigators would recommend oppressively 54 Gy for benign meningiomas, following incomplete resection and upto $60 \mathrm{~Gy}$ for meningiomas that have atypical or malignant features. The postoperative period of this patient was uneventful and after surgery was planned for radiotherapy. After 6 doses, she is doing well till the report.

\section{Conclusion}

Rhabdoid meningiomas are usually encountered in higher grade meningiomas, seen to emerge as a recurrent growth during the process of malignant transition, are seen infrequently in pure form, and have poor outcome. Hence close follow up and aggressive treatment is mandatory in such cases.

\section{References}

1. Weidner N, Cote RJ, Suster S, Weiss LM (2009) Modern Surgical Pathology Vol: 2, 2nd Edition: Central Nervous System Tumors: 2020

2. Haas JE, Palmer NF, Weinberg AG, Beckwith JB (1981) Ultrastructure of malignant rhabdoid tumor of the kidney. A distinctive renal tumor of children. Hum Pathol 12: 646-657.

3. Rorke LB, Packer RJ, Biegel JA (1996) Central nervous system atypical teratoid/rhabdoid tumors of infancy and childhood: definition of an entity. $J$ Neurosurg 85: 56-65

4. Fuller CE, Pfeifer J, Humphrey P, Bruch LA, Dehner LP, et al. (2001) Chromosome 22q dosage in composite extrarenal rhabdoid tumors: clonal evolution or a phenotypic mimic? Hum Pathol 32: 1102-1108.

5. Kepes JJ, Moral LA, Wilkinson SB, Abdullah A, Llena JF (1998) Rhabdoid trahnsformation of tumor cells in meningiomas: a histological indication of increased proliferative activity. Report of four cases. Am J Surg Pathol 22: 231238.

6. Perry A, Scheithauer BW, Stafford SL, Abell-Aleff PC, Meyer FB (1998) "Rhabdoid" meningioma: an aggressive variant. Am J Surg Pathol 22: 1482 1490 .

7. Burger RC (2002) Surgical pathology of the nervous system and its conveings - 4th edition, Intracranial meninges: 61-62.

8. Hojo H, Abe M (2001) Rhabdoid papillary meningioma. Am J Surg Pathol 25 964-969. 